\title{
Hemoglobina glicada A1c no diabetes
}

Leylane Bernardes Forte ${ }^{1}$, Virginia Oliveira Fernandes ${ }^{2}$, Paulo Cruz de Queiroz ${ }^{3}$, Manuela Montenegro Dias de Carvalho ${ }^{3}$, Daniel Duarte Gadelha ${ }^{3}$, Renan Magalhães Montenegro Junior ${ }^{4}$.

1 Residente em Clínica Médica, Hospital Universitário Walter Cantídio (HUWC), Fortaleza, Ceará, Brasil. 2 Doutorado em Ciências Médicas, Universidade Federal do Ceará (UFC), Fortaleza, Ceará, Brasil. 3 Endocrinologista, Hospital Universitário Walter Cantídio (HUWC), Fortaleza, Ceará, Brasil. 4 Doutorado em Clínica Médica, Gerente de Ensino dos Hospitais Universitários da Universidade Federal do Ceará (UFC), Fortaleza, Ceará, Brasil.

\section{Importância e Indicações}

- Hemoglobina glicada (A1c) é o melhor parâmetro preditor de complicações crônicas do Diabetes Mellitus;

- Fundamental na avaliação do controle glicêmico do paciente com diabetes durante seguimento;

- Atualmente também estabelecida para diagnóstico de Diabetes Mellitus (Quadro 1);

- Hemoglobina glicada e Glicohemoglobina (A1c) são as terminologias hoje mais utilizadas.

\section{Princípios do Método}

- Glicação não enzimática de proteína (hemoglobina);

- Formada pela ligação da glicose ao aminoácido valina da porção N-terminal da cadeia beta da hemoglobina por meio de uma ligação estável e irreversível.

\section{Métodos Recomendados}

- São métodos amplamente disponíveis atualmente, padronizados pelo National Glycohemoglobin Standardization Program (NGSP) - www.ngsp.org

- Cromatografia líquida de alta performance (HPLC) por troca iônica;

- Cromatografia por afinidade ou afinidade por boronato;

○ Imunoensaio de inibição turbidimétrica.

\section{Interpretação Clínica}

- Reflete a média de glicemias dos últimos 3-4 meses, sendo:

- $50 \%$ do seu valor referente às glicemias do último mês;

- $25 \%$ dos 2 meses antes da coleta;

- $25 \%$ dos 3-4 meses que antecedem a coleta.

- Como representa apenas o nível médio da glicemia, é fundamental ser associada a automonitoramento glicêmico capilar, com glicosímetros portáteis e/ou monitoramento de glicose intersticial (sensores de medição contínua).

Coleta da Amostra para Determinação da A1c

- Dosada em sangue total, deve ser coletada em tubo com anticoagulante EDTA (tampa roxa);

- Tem estabilidade consideravelmente maior do que a glicemia de jejum;

- O jejum não é necessário para sua coleta, podendo ser efetuada em qualquer horário;

- A amostra é estável em temperatura ambiente por até 24 horas e até 7 dias sob refrigeração.

\section{Interpretação dos Resultados}

Quadro 1. Critérios laboratoriais, segundo a Sociedade Brasileira de Diabetes, para diagnóstico de pré-diabetes e diabetes mellitus, baseados na hemoglobina glicada (A1c).

\begin{tabular}{|l|l|}
\hline & A1c (\%) \\
\hline Normal & $<5,7$ \\
\hline Pré-diabetes & $\geq 5,7<6,5$ \\
\hline Diabetes & $\geq 6,5$ \\
\hline
\end{tabular}


Quadro 2. Metas de controle metabólico baseados na hemoglobina glicada (A1c).

\begin{tabular}{|l|l|}
\hline & A1c (\%) \\
\hline SBD & $<7,0$ \\
\hline ADA & $<7,0$ \\
\hline IDF & $<7,0$ \\
\hline AACE & $<6,5$ \\
\hline
\end{tabular}

SBD: Sociedade Brasileira de Diabetes; ADA: American Diabetes Association; IDF: International Diabetes Federation; AACE: American Association of Clinical Endocrinologists.

\section{Interferentes da A1c}

- Algumas situações clinicas podem levar a falsos valores da A1c (Quadro 3)

Quadro 3. Interferentes da A1c.

\begin{tabular}{|l|}
\hline Falsa elevação da A1c \\
\hline Hipertrigliceridemia grave \\
\hline Alcoolismo crônico \\
\hline Hiperbilirrubinemia \\
\hline Ingestão crônica de salicilatos (3 a $6 \mathrm{~g} /$ dia) \\
\hline Anemia ferropriva \\
\hline Insuficiência renal \\
\hline Toxicidade por opióides \\
\hline Falsa diminuição da HbA1c \\
\hline Anemias hemolíticas \\
\hline Estados hemorrágicos \\
\hline Vitaminas C e E em altas doses (1g/dia) \\
\hline Transfusão de sangue recente \\
\hline Algumas hemoglobinopatias (a depender do método utilizado) \\
\hline
\end{tabular}

\section{Outros marcadores utilizados na avaliação do controle glicêmico}

- Frutosamina:

- nome genérico dado a todas as proteínas glicadas

- $80 \%$ composta por albumina glicada

- representa a média de glicemias ao longo de 2 semanas

- Albumina glicada:

- representa a média de glicemias em 2 semanas

- 1,5-anidroglucitol (1,5-AG):

- proposto para avaliação da variabilidade glicêmica

- $\quad$ se correlaciona inversamente com excursões glicêmicas de curto prazo (72h)

- Tais métodos não estão padronizados para uso amplo na prática clínica, sem valores de referência com estabelecidos com associação a complicações crônicas do Diabetes Mellitus

\section{REFERÊNCIAS}

1 American Diabetes Association. Standards of medical care in diabetes - 2018 abridged for primary care providers. Clin Diabetes. 2018;36(1):14-37.

2 Oliveira JE, Montenegro RM Junior, Vencio S, organizadores. Diretrizes da Sociedade Brasileira de Diabetes 2017-2018. São Paulo: Editora Clannad; 2017. Ciclos de vida e situações especiais, p. 90-93.

Como citar:

Forte LB, Fernandes VO, Queiroz PC, Carvalho MM, Gadelha DD, Montenegro RM Junior. Hemoglobina glicada A1c no diabetes. Rev Med UFC. 2019 jan-mar;59(1):79-80. 